\title{
Structure des peuplements et potentiel de domestication de Parkia biglobosa dans la région de Tandjilé-Ouest (Tchad)
}

\author{
Marie Louise Appolinaire AVANA-TIENTCHEU ${ }^{1 *}$, Sincère KEOUNA ${ }^{1}$, \\ Delphine DONGOCK NGUEMO ${ }^{2}$ et Blaise MOUGA MASDEWEL ${ }^{3}$ \\ ${ }^{I}$ Département de Foresterie, Faculté d'Agronomie et des Sciences Agricoles, Université de Dschang, \\ B.P. 222 Dschang, Cameroun. \\ ${ }^{2}$ Département des Sciences Biologiques, Faculté des Sciences, Université de Ngaoundéré, Cameroun. \\ ${ }^{3}$ Chef de Division des Forêts, Direction des Forêts, Tchad. \\ *Auteur correspondant ; E-mail : avanatie@yahoo.fr
}

\section{RESUME}

Parkia biglobosa, communément appelé néré est la troisième espèce ligneuse la plus importante au Tchad. Cependant, l'absence d'information fiable sur la dynamique de ses peuplements constitue une contrainte qui limite l'élaboration des stratégies pour sa gestion participative et durable. La présente étude avait pour objectif d'évaluer son potentiel écologique et ethnobotanique dans l'un de ses bassins de production au Sud du Tchad. L'approche méthodologique a consisté en des enquêtes ethnobotaniques et inventaires dans les systèmes de production de Parkia biglobosa. Un effectif de 367 tiges a été inventorié à raison de 51,65\% dans les champs agricoles, $34,17 \%$ dans les jachères et $9,17 \%$ dans les forêts claires, soit une densité moyenne de $22,7 \pm 6,11$ arbres/hectare ${ }^{-1}$. La structure des peuplements montre une faible représentation des tiges d'avenir dans tous les types de systèmes. Les organes couramment utilisés sont les graines (52\%) et la pulpe des fruits (32\%) avec des valeurs d'usage essentiellement alimentaires et commerciales. La répartition du potentiel de $P$. biglobosa en faveur des systèmes cultivés traduit l'intérêt des populations pour la domestication de l'espèce. L'étude recommande donc une exploitation rationnelle des fruits, une sensibilisation des populations aux pratiques de production, de gestion et de conservation des plantules en champs.

(C) 2019 International Formulae Group. All rights reserved

Mot clés: Contrainte de gestion, enquête ethnobotanique, Parkia biglobosa, structure des peuplements, valeur d'usage, Tchad.

\section{Stand structure and domestication potentials of Parkia biglobosa in the Tandjilé-West region (Chad)}

\begin{abstract}
Parkia biglobosa, commonly known as néré is the third most important tree species in Chad. Even though there is lack of information on the evolution of natural stands limit the elaboration of a strategy for its sustainable and participative management. A study was conducted in the region of West-Tandjilé with the aim of assessing the ecological and ethnobotanical potentials of this specie. The methodological approach consisted of ethnobotanical surveys and inventories of néré trees in different production systems. A total of 367 stems, distributed in crop farms $(51.65 \%)$, fallow's lands $(34.17 \%)$ and open forests $(9.17 \%)$ was recorded with an
\end{abstract}


average density of $22.7 \pm 6.11$ trees $\mathrm{ha}^{-1}$. The population structure showed an old orchard with a low representation of saplings in all the production systems. The most exploited parts of $P$. biglobosa in the study zone were seeds $(52 \%)$ and fruit pulp (32\%) mainly used as food $(31 \%)$ and for commercialization $(27 \%)$. Overexploitation (43\%), forest carbonization (27\%) and usage conflicts (18\%) were reported as main constraints for sustainable management of the species. The results reveal that the ecologic and socioeconomic potentials of $P$. biglobosa in the study zone were in contrast with the multiplicity of constraints affecting its resources reconstitution. However, the distribution of the tree mainly in cultivated systems indicate farmer's interest for the domestication of the species. In support to this management strategy, the study recommends rational exploitation of fruits, a sensitization on good practices for production, management and conservation of $P$. biglobosa seedlings on farms.

(C) 2019 International Formulae Group. All rights reserved

Keywords: Chad, ethnobotanical survey, management constraints, Parkia biglobosa, population structure, use values.

\section{INTRODUCTION}

Les massifs forestiers jouent un important rôle dans le processus de maintien de la diversité biologique, la stabilisation des bassins hydrologiques et de conservation des sols (FAO, 2014). Ces multiples fonctions ont suscité depuis les années 80 au sein de la communauté internationale un regain d'intérêt pour ces écosystèmes. Cependant, le marasme économique et la pauvreté qui prévalent dans la plupart des pays ont entrainé une pression sans cesse accrue sur les ressources forestières engendrant une déforestation intense qui prive les populations locales d'importantes sources de subsistance et de revenus et qui menace l'existence de milliers d'espèces animales et végétales (Codja et al., 2003 ; Chupezi et al., 2009). Une évaluation récente montre que de nombreuses espèces sont menacées de disparition dans les forêts d'Afrique Centrale parmi lesquelles environ $55 \%$ des espèces végétales dont $10 \%$ déjà éteintes à l'état sauvage (Maréchal et al., 2014). Les défis de la science et des politiques sont de rechercher des stratégies d'aménagement durable et de conservation des écosystèmes forestiers, tout en tenant compte des besoins humains. Dans les zones soudano-sahéliennes, les arbres et arbustes jouent un rôle capital dans la conservation ou la reconstitution de l'équilibre écologique face à l'extension du désert (Maazou et al., 2017). Outre ce rôle primordial, les arbres constituent la principale source des biens et services indispensables aux populations (Eba'a et al., 2013).
Cependant, ces ressources subissent de nombreuses pressions liées essentiellement à la forte démographie, aux variations climatiques et aux systèmes d'utilisation des terres (Guedje et al., 2010 ; Maponmetsem et al., 2011). Une gamme de Produits Forestiers Non Ligneux (PFNL) se distingue au Tchad, parmi lesquels les plus importantes sont la gomme arabique (Acacia senegal L. willd), le karité (Vitellaria paradoxa C.F. Gaertn), le rônier (Borassus aethiopum Mart.) et le néré (Parkia biglobosa Jacq. Benth) (Maponmetsem et Dona, 2010). Cette dernière espèce est un arbre nourricier de la famille des Mimosacées considéré depuis le début des années 2000 comme protégée au Tchad et largement répandue dans les champs et les forêts claires des régions soudaniennes du pays (Blench, 2003). C'est une espèce multifonctionnelle dont l'importance économique, socio-culturelle, médicinale, commerciale et agroforestière a été largement documentée en Afrique de l'Ouest (Bayala et al., 2005 ; Koura et al., 2013 ; Maisharou et Larwanou, 2015). L'espèce est principalement valorisée pour ses graines fermentées qui sont transformées en «dawa-dawa» ou moutarde africaine, produit très nutritif et très prisé pour relever le goût de diverses sauces et mets dans de nombreux pays sahéliens (Azokpota et al., 2006). De même, la pulpe des graines du néré est consommée directement ou associée à la farine de blé, du maïs ou du petit mil pour faire des beignets (Moulnang et Kadiom, 2001). P. biglobosa constitue également une 
ressource importante pour la population du Département de la Tandjilé Ouest qui en est totalement dépendante pour la satisfaction de leurs besoins quotidiens (alimentaire, médicinal, énergétique, culturel, rituel). Cependant, peu d'études ont jusqu'ici été consacrées à l'évaluation ethnobotanique et écologique de l'espèce dans la région de la Tandjilé en particulier et au Tchad en général. Pourtant, la disponibilité d'informations sur l'état actuel du peuplement, les types d'usage du néré parait indispensable pour permettre de mieux contribuer à sa gestion durable (Nchoutpouen et al., 2009 ; Maponmetsem et al., 2011; Koura et al., 2013 ; Padakale et al., 2015). A ce titre, des travaux ont montré qu'une meilleure documentation de la valeur accordée par les communautés à une espèce constitue le point de départ pour son utilisation durable et pour l'élaboration d'une stratégie raisonnée de conservation (Nchoutpouen et al., 2009 ; Maponmetsem et al., 2011; Dicko et al., 2017). L'objectif de cette étude est de contribuer à la gestion durable et à la valorisation du néré au Tchad. Plus spécifiquement, il s'agit d'évaluer (1) la structure des peuplements à néré dans les différents systèmes de production, (2) les types d'organes et les formes d'usage, (3) les contraintes à la gestion durable de l'espèce dans quatre villages de la région de TandjiléOuest.

\section{MATERIEL ET METHODES \\ Description de la zone d'étude}

Le Département de la Tandjilé Ouest s'étend sur une superficie de $12345 \mathrm{~km}^{2}$ et est globalement compris entre $9^{\circ} 12^{\prime}$ de longitude Est et $15^{\circ} 42^{\prime}$ de latitude Nord (Figure 1). La population est estimée à 527340 habitants selon le Recensement General de la Population et de l'Habitat (RGPH2) effectué en 2009. Le climat est de type sahélo soudanien et le relief est dominé par des zones exondées et inondables. Les plaines de la région de la Tandjilé sont les zones temporairement inondées par les pluies directes accompagnées de débordements fluviaux. Ce sont des zones inter fluviales décrites selon la carte des ressources en eau souterraine de l'Unicef, comme ayant une profondeur faible de la nappe phréatique.

Les sols sont qualifiés comme bons et propices à presque toutes les variétés de cultures pratiquées dans la région. $\mathrm{La}$ végétation est très variée et se caractérise par quatre (4) types d'écosystèmes à savoir : la forêt claire ; la savane ; la steppe ; la prairie. Le régime pluviométrique est celui des climats de type soudanien caractérisé par une saison sèche (novembre à mars) et une saison pluvieuse (avril à octobre). Les forêts claires et les savanes sont dominées par plusieurs espèces ligneuses dont les plus recherchées sont Parkia biglobosa, Vitellaria paradoxa, Tamarindus indica et Borassus akeassii. Les Marba, Lélé et Gambaye sont les ethnies dominantes de la région qui comporte six sous-préfectures : Dafra, Dogou, Kolong, Bologo, Baktchoro et Kelo (Maponmetsem et Dona, 2010).

\section{Méthodologie}

Quatre villages appartenant aux souspréfectures de Bologo et Dogou dans la région de la Tandjilé-Ouest ont été choisis comme sites d'étude en raison de la forte implication de leurs populations dans l'exploitation du Néré. Dans chacun de ces villages, les enquêtes ont été effectuées dans les ménages et les inventaires sur le terrain.

\section{Enquêtes ethnobotaniques dans les ménages}

Dans ces villages, un questionnaire semi-structuré et un guide d'entretien ont servi d'outils de collecte des données auprès de 285 répondants dont 196 femmes et 89 hommes répartis comme suit: autorités traditionnelles et administratives (14.5\%), producteurs et/ou consommateurs (42.1\%), commerçants $(43.5 \%)$. L'âge des enquêtés variait entre 15 et 93 ans. Des groupes de discussion avec les populations suivis des observations directes sur le terrain ont permis d'identifier les types de système de production, soit environ 31 enquêtés par village en dehors des autorités administratives. Les enquêtes individuelles auprès des collecteurs, producteurs, commerçants et consommateurs, à raison de 60 répondants par village, ont porté sur les 
types de produits, les formes et les valeurs d'usages ainsi que les contraintes pour la gestion durable de la ressource (Houehanou et al., 2016).

\section{Evaluation du potentiel sur pied et de la structure des arbres à néré dans les sites d'étude}

Dans chaque village, l'identification des parcs à néré a été faite de façon raisonnée avec l'aide des chefs de village. La méthode d'inventaire des arbres dans les parcs identifiés s'est inspirée du manuel pour le relevé intégré de données sur le terrain de l'Organisation des Nations Unies pour l'Alimentation et l'Agriculture (FAO, 2009) ainsi que des travaux antérieurs effectués sur la même espèce et sur le karité au Nord du Cameroun (Nchoutpouen et al., 2009; Maponmetsem et al., 2011). Au total, 16 unités de comptage (100 m x $100 \mathrm{~m})$ ont été implantées dans le site, à raison de deux unités de comptage par village. Chacune des unités de comptage a été divisée en 2 placettes rectangulaires de $100 \mathrm{~m} \times 50 \mathrm{~m}$, soit 32 placettes prospectées pour l'ensemble de la zone d'étude. Toutes les tiges d'arbres à néré de diamètre supérieur à $10 \mathrm{~cm}$ ont été inventoriées, géo-référencées et leur diamètre à $1,3 \mathrm{~m}$ du sol a été mesuré à l'aide d'un ruban forestier. La mesure de la hauteur de ces arbres a été faite selon la méthode détaillée de la croix du bûcheron.

\section{Analyse statistique}

Les principales réponses obtenues sur les organes exploités et leurs usages ont permis d'évaluer la valeur d'usage (UVs) de l'espèce (Houehanou et al., 2016). Les catégories d'usages relatives à chaque organe de $P$. biglobosa ont été déterminées à partir des fréquences d'utilisation selon la formule :

$$
U V_{s}=\frac{\sum_{i}^{n} U_{i s}}{n_{s}}
$$

$\left(\mathrm{n}_{\mathrm{s}}:\right.$ nombre

total d'informateurs interviewés sur l'espèce $\mathbf{s}$ ; $\mathbf{U}_{i s}$ : nombre d'usages selon l'informateur i).

La fréquence Relative de citations (FRC) révèle l'importance de chaque organe/usage et est calculée sur la base des fréquences de citations (FC) en utilisant la formule :

$$
\mathrm{FRCs}=\mathrm{FCs} / \mathrm{N}
$$

La valeur de FC est divisée par le nombre total de répondants ayant participé aux enquêtes.

Ces données ethnobotaniques ont été soumises à une analyse de contenu, puis aux statistiques descriptives. Les valeurs d'usages et les fréquences relatives des citations ont été comparées entres les villages/ethnies et les catégories d'usages à travers une analyse de variance. Les différences des opinions sur les contraintes de gestion durable de l'espèce ont été comparées en fonction du genre des répondants (femmes, hommes, jeunes et vieux) en utilisant le test de Khi deux.

Les données dendrométriques et structurales collectées ont été utilisées pour évaluer les paramètres suivants:

- La surface terrière moyenne $(\mathrm{g}): \mathbf{g}=\frac{\mathbf{m D}^{\mathbf{2}}}{\mathbf{4}}(\mathrm{g}=$ Surface terrière de l'arbre $\left(\mathrm{m}^{2} / \mathrm{ha}\right) ; \mathrm{D}=$ Diamètre des arbres).

- La densité $(\mathrm{N})$ est exprimée par: $\mathrm{N}=\mathrm{n} / \mathrm{S}(\mathrm{N}=$ densité (Néré/ha), $\mathrm{n}=$ nombre total d'individus; $\mathrm{S}=$ aire totale échantillonnée dans le village (ha)).

- La surface terrière totale $(\mathrm{G})$ $=$ somme des surfaces terrières des arbres pris individuellement qui composent le peuplement:

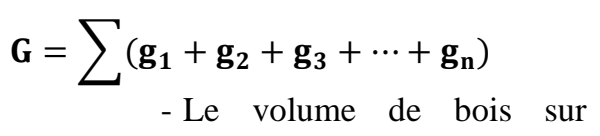
pieds (V) a été évalué en se basant sur la formule suivante :

$$
\mathrm{V}=\mathrm{G} \times \mathrm{Ht} \text { x f }(\mathrm{G}=\text { surface terrière }
$$

$\left(\mathrm{m}^{2}\right), \quad \mathrm{Ht}=$ hauteur totale $(\mathrm{m}), \mathrm{f}=$ coefficient de forme $=0,65$ ) (Ræbild et al., 2012).

Les données de diamètre des tiges inventoriées ont permis de les répartir par classes de diamètre d'amplitude $10 \mathrm{~cm}$. Les densités d'arbres pour les différentes classes de diamètre ont été déterminées pour les différents systèmes de production. Ces données ont été utilisées pour élaborer des 
histogrammes de distribution des densités des arbres de néré par classe de diamètre. Les courbes d'ajustement ont été élaborées pour mieux rendre compte de l'état des peuplements de $P$. biglobosa dans les différents systèmes de production en utilisant le Logiciel EXCEL 2016. Les caractéristiques dendrométriques et structurales de $P$. biglobosa ont été comparés au sein du même système de production et entre les systèmes grâce au test ANOVA effectué à l'aide du logiciel XLSTAT 2014 et les moyennes significativement différentes ont été séparées en utilisant le test de Student Newman and Keuls (SNK).

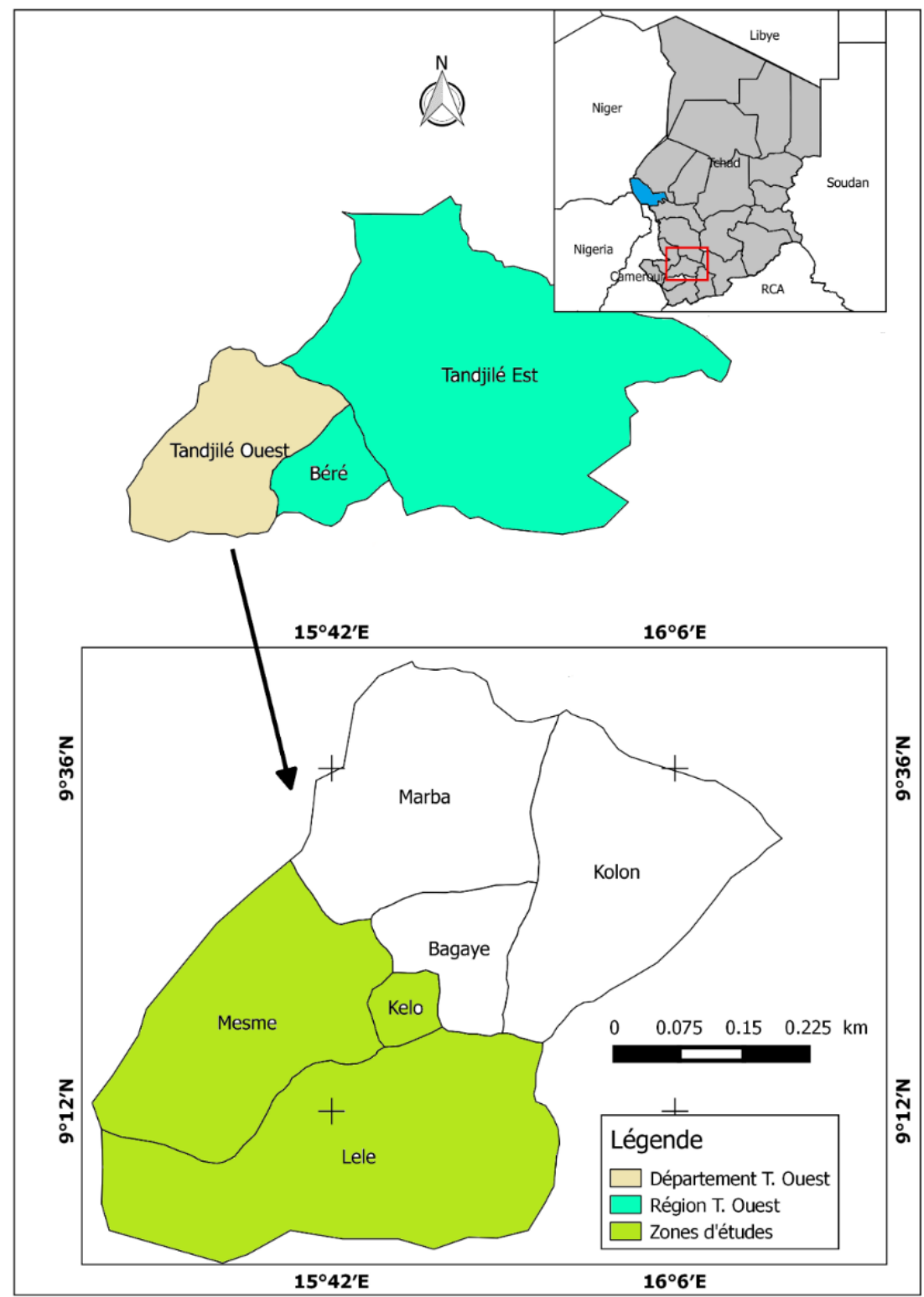

Figure 1: Carte de distribution des arbres à néré dans les quatre localités échantillonnées à Tandjilé-Ouest, Tchad. 


\section{RESULTATS}

\section{Distribution spatiale et systèmes de production de néré}

L'arbre à néré est retrouvé dans quatre types de systèmes de production dans la zone de Tandjilé-Ouest parmi lesquels les champs agricoles $(51,67 \%)$ et les jachères $(34,17 \%)$ sont les plus dominants par rapport aux forêts claires et jardins de case (Figure 2). Un total de 367 individus de néré a été inventorié sur une superficie de 16 ha répartie dans trois des systèmes de production que sont les champs agricoles, les jachères et les forêts claires. Le potentiel sur pied est plus élevé dans les champs agricoles avec 183 individus pour toutes les quatre localités échantillonnées avec un nombre moyen d'arbres par localité variant de 10,7 $\pm 0,58$ à $30 \pm 5,66$ à Bongor Baou et Mangsou Yaougué respectivement. Dans les jachères, 148 arbres ont été inventoriés dans trois des quatre localités, soit Mangsou Yaougué (27 $\pm 1,41$ arbres), Dissoua Tamssitoro (28 $\pm 5,7$ arbres) et Bongor Gaourang (38 arbres).

Les forêts claires ont été observées à Bongor Baou (9 individus) et Bongor Gaourang (27 individus) (Tableau 1). Les effectifs totaux de tiges recensées par localité varient de 40 à Bongor Baou, 97 à Dissoua Tamssitoro, 114 à Mangsou Yaougué et 116 à Bongor Gaourang, soit un effectif moyen de $92 \pm 35,5$ arbres par village.

\section{Structure des peuplements du néré}

Les caractéristiques structurales résumées au Tableau 2 montrent que les diamètres moyens des arbres varient de $62,97 \pm 16,53 \mathrm{~cm}$ dans les jachères à $73,92 \pm 14,10$ dans les champs agricoles. La hauteur totale moyenne des arbres varie très peu entre les trois systèmes étudiés avec des valeurs comprises entre $12,54 \mathrm{~m}$ (forêts claires) et 14,68 m (jachères). La surface terrière et la densité moyenne varient de 6,40 à $12,56 \mathrm{~m}^{3} /$ ha et de $18 \pm 12,73$ à $28 \pm 5,7$ arbres/ha pour les systèmes forêts claires et champs agricoles respectivement. L'analyse de variance montre que ces paramètres ne sont pas significativement influencés par le type de système de production malgré les écarts observés entre les valeurs ( $p>0,05$ ). Le volume sur pied est le seul paramètre qui est significativement plus élevé dans les champs agricoles $\left(215,02 \mathrm{~m}^{3}\right)$ et les jachères $(210,79$ $\left.\mathrm{m}^{3}\right)$ que dans les forêts claires $\left(96,39 \mathrm{~m}^{3}\right)(\mathrm{P}=$ $0,0010)$.

La structure en diamètre des arbres à néré présente une distribution erratique dans tous les systèmes de production étudiés (les champs, les forêts claires et les jachères). De manière générale, une forte densité de tiges est observée dans les classes de diamètre [30 40[et [90 - 100[. Dans les champs agricoles, ces deux classes sont représentées respectivement par 22 et 23 pieds/ha (Figure $3 \mathrm{~A})$. Les jachères sont dominées par les individus de la classe de diamètres [30 - 40[ avec une densité de 27 tiges/ha (Figure 3B). Dans les forêts claires, les peuplements de $P$. biglobosa sont plus vieillissants avec une forte représentation des tiges dans la classe de [50 60[ qui décroît progressivement lorsque le diamètre augmente (>130) (Figure 3C). Dans tous les systèmes de production, la structure des arbres s'ajuste par une équation polynomiale avec des valeurs de coefficients de détermination $\mathrm{R}^{2}$ de 0,74 pour les champs agricoles et les jachères et de 0,58 pour les forêts claires. Une telle distribution irrégulière est caractéristique des systèmes perturbés.

La structure en hauteur des peuplements de néré montre également une distribution en cloche avec une forte représentation des arbres dans le centre des classes [10-15] m. L'équation polynomiale ajustée montre, pour tous les systèmes de production étudiés, des valeurs de coefficients de détermination $\left(\mathrm{R}^{2}\right)$ supérieures 0,92 (Figure 4.A, B et C).

\section{Formes et valeurs d'usage du Néré}

Les résultats du Tableau 3 montrent que sept formes d'usage du néré ont été citées par les populations de la zone d'étude. Parmi ces usages, l'alimentation (31\%), la commercialisation des produits $(27 \%)$ et l'utilisation comme bois de chauffage (22\%) sont les plus fréquemment cités. Cependant, les fonctions alimentaires et de sources 
d'énergie familiale sont considérées comme les plus importantes car elles jouent un rôle primordial pour la survie des populations surtout en période de disette et de pénurie d'énergie alternative.

Il ressort également des résultats d'enquête que la graine (52\%), la pulpe des fruits $(28 \%)$ et l'écorces (9\%) sont par ordre d'importance décroissante des fréquences relatives de citations (FRCs) les organes de $P$. biglobosa les plus exploités dans la zone d'étude et utilisés essentiellement pour l'alimentation (graines et pulpe) et la médecine traditionnelle (écorce) (Figure 5). En effet, la pulpe des fruits et les graines sont largement consommées sous différentes formes par les populations. Les feuilles, l'écorce et la racine entrent dans le traitement de plusieurs maladies parmi lesquelles: la méningite, la stérilité, les bronchites, les trachéides, la pneumonie, les infections sexuellement transmissibles (IST), la lèpre, le rachitisme, les coliques, la diarrhée, l'ostéopathie, les oreillons, les dermatoses, les filarioses, les œdèmes, les bronchites, les brûlures et les hémorroïdes. Les feuilles sont également utilisées comme fourrage. En plus du bois énergie et de la carbonisation, le bois de $P$. biglobosa est utilisé dans la fabrication des meubles et des objets d'art.

\section{Opinion des enquêtés sur les contraintes de gestion durable du néré}

Quatre principales contraintes ont été signalé par 32\% des enquêtés pour la gestion des arbres à néré dans les agrosystèmes soit: le prélèvement abusif des organes (43\%), la carbonisation de la forêt pour la production du charbon de bois (27\%), les conflits d'usage $(18 \%)$, les feux de brousse (12\%) et les pluies violentes (9\%). Le test de $\mathrm{khi}^{2}\left(\chi^{2}=4,671 ; \mathrm{P}=\right.$ $0,031)$ montre une différence significative entre les perceptions des répondants indiquant que le niveau de connaissances et les opinions des enquêtés sur les contraintes varient en fonction du sexe $(\mathrm{P}=0.000)$ et de l'âge des répondants $(\mathrm{P}=0,002)$. $\mathrm{La}$ forte pression exercée sur les organes des végétaux serait dues pour des raisons non seulement alimentaires mais également médicinales, en effet la population de ces zones tire l'essentiel de leur besoin de leur écosystème.

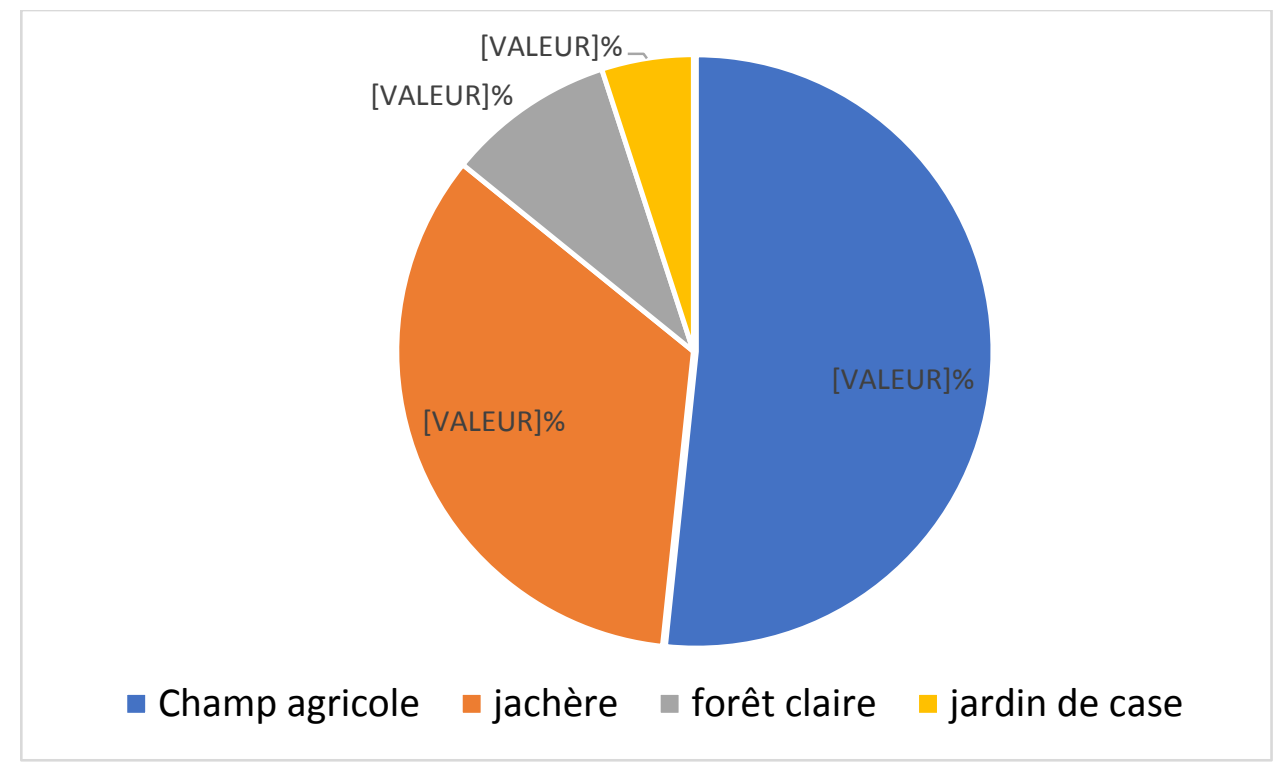

Figure 2 : Types de systèmes de production de Parkia biglobosa 


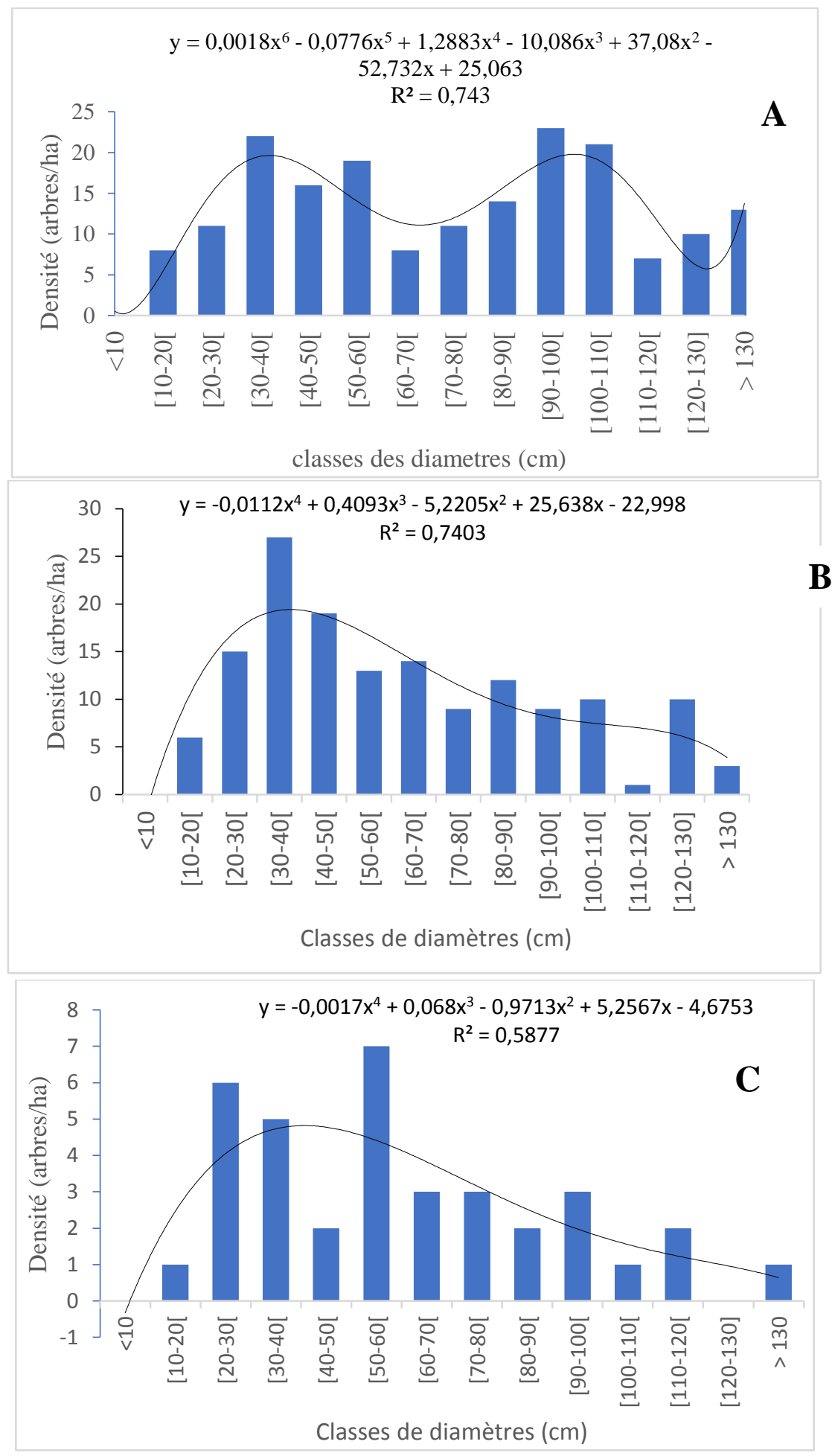

Figure 3 : Distribution de tiges par classes de diamètres dans les champs agricoles (A), les jachères (B) et les forêts claires (C). 

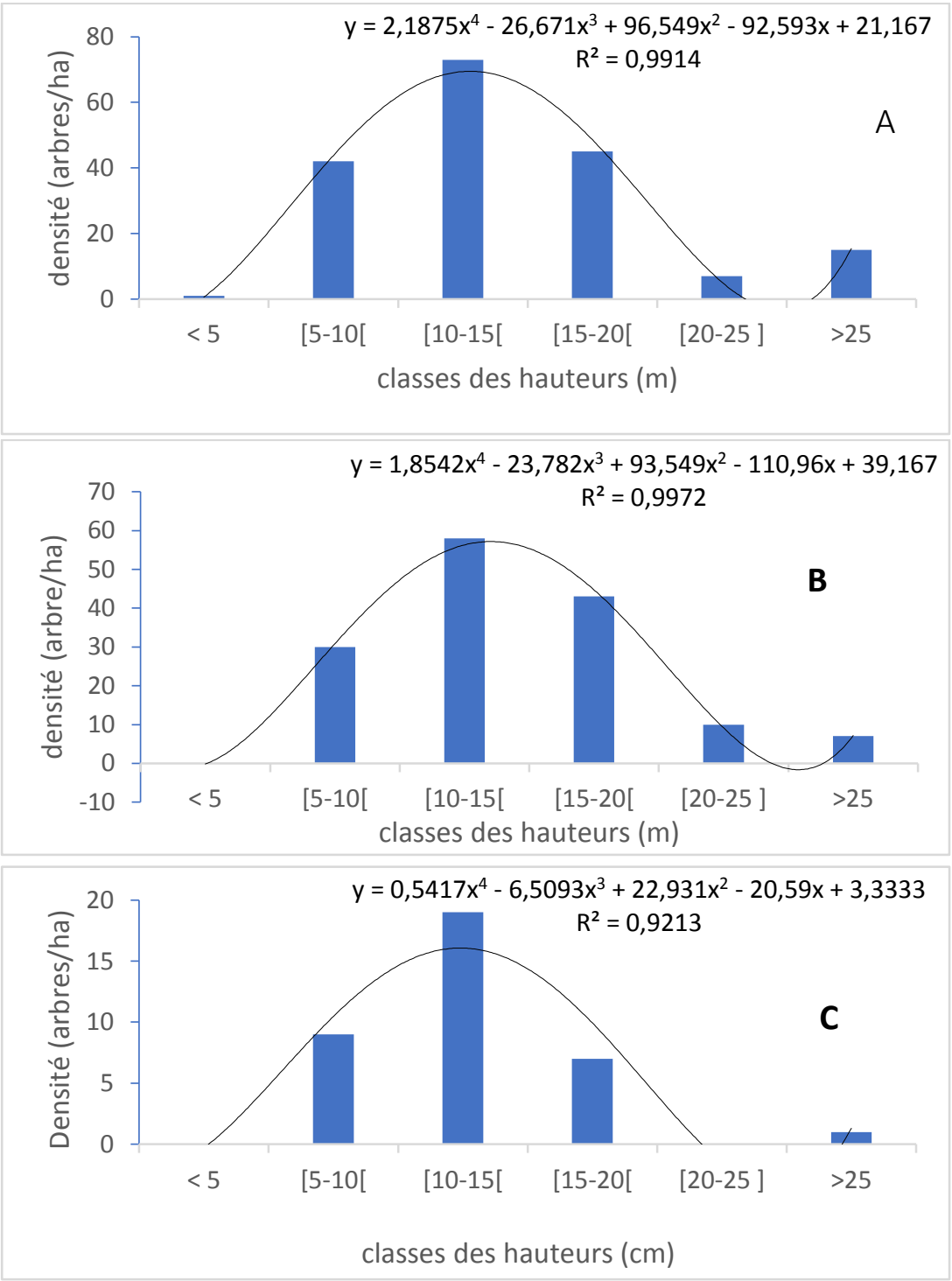

Figure 4 : Distribution des arbres de Parkia biglobosa par classe de hauteurs dans les Champs agricoles (A), les jachères (B) et les forêts claires (C).

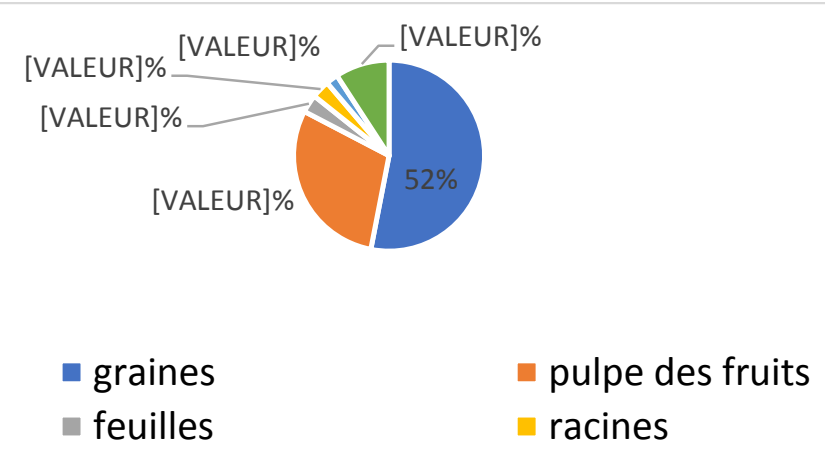

Figure 5 : Fréquences relatives des citations pour les différents organes de Parkia biglobosa utilisés par les populations de la région. 
Tableau 1. Nombre moyen de tiges de Parkia biglobosa dans les différents systèmes de production en fonction des localités.

\begin{tabular}{clcc}
\hline \multirow{2}{*}{ Localités } & \multicolumn{3}{c}{ Systèmes de production de P. biglobosa } \\
\cline { 2 - 4 } & Champs agricoles & jachères & Forêts claires \\
\hline MY & $30 \pm 5,66$ & $27 \pm 1,41$ & 0 \\
BB & $10,67 \pm 0,58$ & 0 & 9 \\
BG & $25,5 \pm 9,2$ & 38 & 27 \\
DT & $20,5 \pm 2,13$ & $28 \pm 5,7$ & 0 \\
\hline
\end{tabular}

MY : Mangsou Yaougué ; BB : Bongor Baou ; BG : Bongor Gaourang, et DT : Dissoua Tamssitoro.

Tableau 2. Paramètres dendrométriques et structuraux de $P$. biglobosa dans les systèmes de production.

\begin{tabular}{cccccc}
\hline Paramètres & $\begin{array}{c}\text { Champs } \\
\text { agricoles }\end{array}$ & Jachères & $\begin{array}{c}\text { Forets } \\
\text { claires }\end{array}$ & P & Significativité \\
\hline Effectif de tiges & 183 & 148 & 36 & & Ns \\
\hline Diamètre (cm) & $73,92 \pm 14,10^{\mathrm{a}}$ & $62,97 \pm 16,53^{\mathrm{a}}$ & $67,95 \pm 22.08^{\mathrm{a}}$ & 0,4906 & $\mathrm{Ns}$ \\
\hline Hauteur $(\mathrm{m})$ & $13,79 \pm 2,81^{\mathrm{a}}$ & $14,68 \pm 3,47^{\mathrm{a}}$ & $12,64 \pm 0.69^{\mathrm{a}}$ & 0,702 & $\mathrm{NS}$ \\
\hline Densité (tige/ha) & $20,44 \pm 8,96^{\mathrm{a}}$ & $29,6 \pm 5,50^{\mathrm{a}}$ & $18 \pm 12,07^{\mathrm{a}}$ & 0.4952 & $\mathrm{Ns}$ \\
\hline ST $\mathrm{m}^{2} / \mathrm{ha}$ & $12,56 \pm 7,93^{\mathrm{a}}$ & $11,55 \pm 5,09^{\mathrm{a}}$ & $6,40 \pm 1,44^{\mathrm{a}}$ & 0,530 & $*$ \\
\hline Volume $\left(\mathrm{m}^{3}\right)$ & $215,02^{\mathrm{a}}$ & $210,79^{\mathrm{a}}$ & $96,39^{\mathrm{b}}$ & 0,0010 & $*$ \\
\hline
\end{tabular}

NB : les moyennes sur la même ligne affectées aux mêmes lettres sont semblables alors que celles affectées aux lettres différentes sont significativement différentes au seuil de $5 \%$ d'après ANOVA un facteur, ST= Surface Terrière ; P = probabilité ; ns =non significatif ; * significatif.

Tableau 3: Valeur d'usage accordée aux produits de Néré en fonction des villages.

\begin{tabular}{|c|c|c|c|c|c|c|c|c|}
\hline \multirow[t]{2}{*}{ Villages } & \multicolumn{5}{|c|}{ Formes d'usages } & \multirow[b]{2}{*}{$\mathbf{O}$} & \multirow[b]{2}{*}{ Total } & \multirow[b]{2}{*}{$\begin{array}{l}\operatorname{FRCs}(\%) \\
(P=0,000)\end{array}$} \\
\hline & $\mathbf{A}$ & $\mathbf{M}$ & $\mathbf{R}$ & BC & $\mathbf{U C}$ & & & \\
\hline MY & 16 & 9 & 1 & 9 & 11 & 6 & 52 & $42,98^{\mathrm{a}}$ \\
\hline $\mathrm{BG}$ & 2 & 0 & 0 & 1 & 1 & 1 & 5 & $04,13^{d}$ \\
\hline$\overline{B B}$ & 13 & 3 & 2 & 10 & 12 & 1 & 41 & $33,88^{b}$ \\
\hline DT & 6 & 0 & 1 & 6 & 8 & 1 & 23 & $19,00^{\mathrm{c}}$ \\
\hline Total & 37 & 12 & 4 & 26 & 32 & 9 & & - \\
\hline FRCs (\%) & $30,83^{a}$ & $10^{\mathrm{c}}$ & $3,33^{\mathrm{d}}$ & $21,66^{b}$ & $26,7^{b}$ & $07,5^{\mathrm{c}}$ & & 100 \\
\hline $\mathrm{P}=0,002$ & & & & & & & & \\
\hline
\end{tabular}

A : Alimentation ; M : Médicinal ; R : Rituel ; BC : Bois de Chauffage ; UC : Usage Commercial ; O : Ombrage). MY : Mangsou Yaougué ; BB : Bongor Baou ; BG : Bongor Gaourang, et DT : Dissoua Tamssitoro. 


\section{DISCUSSION}

L'étude a permis d'identifier quatre types de système de production du néré qui sont les forêts claires, les champs agricoles, les jachères et les jardins de case. Les forêts claires regorgent moins de tiges de néré et seuls quelques arbres isolés sont préservés dans les jardins de case. Ces résultats traduisent la volonté des populations de conserver cette espèce ainsi que d'autres telles que Vitellaria paradoxa et Tamarindus indica qui leur sont directement utiles dans leurs systèmes agricoles. Des résultats similaires ont été obtenus par Odebiyi et al. (2004) dans les savanes humides du Nigéria. Au Cameroun, on a observé que près de $80 \%$ du potentiel des arbres à karité sont conservés dans les systèmes naturels (Maponmetsem et al., 2011). Dans le cadre de la présente étude, la forêt claire n'a été mentionnée que dans deux des quatre localités indiquant que les populations de la région de Tandjilé dépendent presqu'exclusivement des arbres à néré préservés dans leurs plantations en culture et en jachère.

\section{Structure de peuplement}

La densité, le diamètre et la hauteur moyens de Parkia biglobosa ne présentent aucune variation significative en fonction des systèmes de production, mais les valeurs les plus élevées ont été observées dans les champs agricoles. Des résultats similaires ont été observés au Sud du Burkina Faso où on a noté que le type de systèmes de culture n'influence pas la densité des individus de P. biglobosa (Ræbild et al., 2012). Cependant, il a été observé au Togo que les pratiques de gestion des arbres par les agriculteurs contribuent à favoriser une croissance plus importante en diamètre dans les champs et en hauteur dans les jachères (Padakale et al., 2015). Les valeurs de densité (18 à 28 pieds/ha) et de hauteur (12 à $14 \mathrm{~m}$ ) observées sont comparables à celles obtenues pour certains peuplements de néré au nord du Bénin et au Nigéria (Odebiyi et al., 2004 ; Koura et al., 2013). Le faible potentiel en $P$. biglobosa des forêts claires dans la zone d'étude pourrait s'expliquer par une surexploitation des tiges dans ce type de système sous représenté dans la région et généralement facile d'accès.

La surface terrière moyenne varie de 6,40 à $12,56 \mathrm{~m}^{3} / \mathrm{ha}$ dans les forêts claires et les champs agricoles respectivement. Bien que ces variations ne soient pas statistiquement significatives, ces résultats traduisent une meilleure représentation de l'espèce dans les systèmes agricoles gérés par les hommes par rapport aux systèmes naturels. De tels résultats traduiraient l'intérêt des populations pour la domestication de l'espèce et devraient être soutenues à travers une sensibilisation et une formation des agriculteurs aux meilleures pratiques de gestion de l'espèce en association avec les cultures. De manière générale, les valeurs de diamètre moyen et de surface terrière pour les peuplements tchadiens de $P$. biglobosa sont supérieures à celles trouvées au Nigéria, au Benin et au Togo (Odebiyi et al., 2004 ; Koura et al., 2013 ; Padakale et al., 2015). Ces résultats pourraient indiquer que les parcs à néré de la région de Tandjilé sont dominés par des arbres à grands diamètres témoignant d'un peuplement plus âgé ou bénéficiant des conditions édaphiques et environnementales plus favorables (Omondi et al., 2016).

La structure en classes de diamètre et de hauteur présente sensiblement la même distribution dans les trois systèmes étudiés. $\mathrm{Ce}$ résultat pourrait indiquer que les conditions de développement et de croissance des arbres à néré sont assez semblables dans ces trois types de milieux malgré les différences d'orientation de gestion. Ainsi, les pratiques des agriculteurs pour l'entretien des arbres dans les différents systèmes ne permettraient pas de modifier significativement leur structure par rapport au système naturel. Ces observations pourraient s'expliquer par le fait que les arbres présents dans les champs et les jachères ont tout simplement été épargnés lors de la conversion des forêts claires en champs de culture. Très peu d'initiatives sont entreprises par les agriculteurs pour la régénération de l'espèce (Maponmetsem et Dona, 2010). En général, la demande en fruits et en graines de $P$. 
biglobosa est très élevée dans les villages et selon les informateurs, presque toutes les gousses sont récoltées après leur maturation, laissant très peu de semences à disséminer dans le paysage. Cette surexploitation des fruits et graines justifie la faible régénération naturelle et donc la faible représentation des tiges d'avenir (10-20 cm de diamètre) dans les différents systèmes inventoriés.

L'allure globalement irrégulière des courbes ajustées à ces structures traduit des peuplements perturbés. Des résultats similaires ont été mentionnés au Nigéria et au Nord du Benin (Odebiyi et al., 2004 ; Koura et al., 2013) où la structure n'obéissait ni à la distribution exponentielle ni à celle en «cloche». Ce qui s'explique par le fait que dans ces milieux, les pressions anthropiques ne laissent pas les processus de régénération aller jusqu'à leur terme. Par contre, les parcs à néré observés dans les jachères et champs agricoles jeunes et âgés du Togo sont dominés par de jeunes tiges, traduisant des méthodes de gestion plus intensives favorisant une préservation des jeunes tiges et une réduction progressive de la densité des tiges âgées pour permettre un bon développement des cultures associées (Padakale et al., 2015). Cette approche de gestion est par ailleurs confirmée par des densités en général plus élevées pour des diamètres et des surfaces terrières plus faibles des parcs à néré togolais comparés à ceux du Bénin, du Nigéria et du Burkina Faso.

\section{Forme et valeur d'usage du Néré}

Les résultats montrent que six types d'organes pour six principales catégories d'usage ont été listés par les populations de la zone d'étude. Ces valeurs sont inférieures à celles obtenues au Bénin où une dizaine de parties de la plante sont valorisées pour huit catégories d'usage (Koura et al., 2011). Ce qui indiquerait un niveau plus élevé de connaissances sur l'espèce au Bénin, et un potentiel supplémentaire pour la valorisation de l'espèce au Tchad à travers une sensibilisation des populations aux nouveaux usages jusqu'ici méconnus. Toutefois, il est à relever que la présente étude n'a pas couvert toute l'aire de distribution de l'espèce au
Tchad. Les travaux antérieurs relèvent que les niveaux de connaissance ethnobotaniques varient et augmentent avec le nombre d'ethnies et de territoires échantillonnés (Reyes-Garcia et al., 2004). Il est probable qu'une étude plus large couvrant la diversité des ethnies et l'aire de distribution de l'espèce au Tchad permettent de documenter plus de formes d'usage et d'organes utilisés.

Les produits du néré les plus exploités par la population enquêtée sont les graines et la pulpe des fruits. Ce qui témoigne de l'importance essentiellement alimentaire de l'espèce dans la zone d'étude. Des observations similaires ont été obtenues dans la zone soudanienne du Moyen-Chari au Tchad (Dongock et al., 2017). Des travaux antérieurs relèvent que les graines fermentées et la pulpe farineuse des gousses riche en sucre sont deux parties de $P$. biglobosa hautement appréciées dans l'alimentation des populations de tous les pays de l'Afrique de l'Ouest (Bellefontaine et al., 2012; Abdourhamane et al., 2017). En effet, les condiments alimentaires issus des graines constitueraient le principal assaisonnement pour les sauces dans la région. Nos résultats montrent que ce savoir-faire sur l'utilisation des graines et fruits de $P$. biglobosa s'étend au-delà de l'Afrique de l'Ouest et traduirait une certaine similitude dans les habitudes alimentaires des populations vivant dans l'aire de distribution de l'espèce.

L'usage médicinal du néré ne représente que $10 \%$ des formes d'usage citées par les populations de Tandjilé. Au Benin, il a été observé que l'usage médicinal du néré était le plus diversifié et le plus dominant (42\%) comparé à l'usage alimentaire (22\%) (Koura et al., 2011). Les propriétés médicinales de $P$. biglobosa ont été confirmées à travers des études microbiologiques et phytochimiques qui révèlent que ces organes sont riches en composés tels que les flavonoïdes, les tanins, les acides gras, les sucres et les protéines (Azokpota et al., 2006 ; Millogo-Kone et al., 2009 ; Komolafe et al., 2014). Nos résultats pourraient indiquer que le potentiel médicinal 
de l'espèce reste sous-estimé dans la zone d'étude.

Les résultats de la présente étude montrent que les savoirs sur les usages du néré varient en fonction des villages et donc des ethnies. Les populations de deux des quatre localités échantillonnées au Tchad (Mangsou Yaougué et Bongor Gaourang) détiennent l'essentiel des connaissances sur les formes et les valeurs d'usage des organes de $P$. biglobosa par rapport à celles de Bongor Baou (BB) et de Dissoua Tamssitoro (DT). Reyes-Garcia et al. (2004) ont montré qu'il y avait une corrélation forte entre le niveau de connaissances locales sur une espèce et son potentiel sur pied dans l'environnement immédiat. Ceci est confirmé dans la présente étude ou le nombre d'arbres recensés était significativement plus élevé dans les localités de Mangsou Yaougué et de Bongor Gaourang comparé aux deux autres localités.

La commercialisation des produits issus de la transformation de néré représente pour les agriculteurs une stratégie pour augmenter et diversifier leurs revenus. Des résultats similaires ont été obtenus au Bénin ou l'usage commercial représente $22 \%$ des formes d'usage citées par les populations (Koura et al., 2011). Les populations de Tandjilé affirment que la filière néré est une activité très porteuse et ayant contribué au changement de leur mode de vie et la moyenne des revenus mensuels obtenus est largement supérieure à celle observée dans d'autres régions sahéliennes (Goudiaby, 2014; Maisharou et Lawarnou, 2015).

Six contraintes ont été relevées pour la gestion durable du $P$. biglobosa par les populations de la zone d'étude parmi lesquelles la surexploitation et les feux de brousse étaient les plus couramment cités. Ces résultats corroborent ceux observés au Cameroun, indiquant que les feux qui passent chaque année ralentissent et entravent le développement des espèces ligneuses préservés dans les champs parmi lesquelles $P$. biglobosa (Nchoutpouen et al., 2009).
Implication pour la conservation de $P$. biglobosa au Tchad

Betti et al. (2011) ont défini une méthode d'évaluation de la vulnérabilité des espèces à produits forestiers non ligneux basée sept critères donc le type biologique, le nombre et le type d'organe collectés et les pratiques d'exploitation, la popularité, le type d'habitat et son statut de conservation, l'état de développement de la plante au moment de l'exploitation et les principales formes d'usage. Une évaluation de $P$. biglobosa sur la base de ces critères indique un niveau de vulnérabilité élevé pour tous les paramètres dans la zone d'étude. En effet, de par son type biologique «arbre», la croissance et le développement de $P$. biglobosa sont contraints par les facteurs évolutifs tels que la sélection naturelle, les flux de gènes, les mutations et les goulots d'étranglement qui fragilisent ses peuplements auxquels s'ajoutent les pressions anthropiques (Omondi et al., 2016a). L'impact de ces pressions est généralement évalué à travers la structure démographique des peuplements (Nchoutpouen et al., 2009; Maponmetsem et al., 2011; Omondi et al., 2016a ; Abdourhamane et al., 2017). Dans le cadre de la présente étude, la structure des arbres à néré montre la quasi-absence des tiges dans certaines classes de diamètre, ce qui témoigne de l'occurrence de ces pressions.

La diversité de produits et d'organes sollicités par les populations augmente la vulnérabilité de $P$. biglobosa. En plus de la multiplicité des organes exploités, les pratiques d'exploitation qui impliquent l'écorçage des tiges et l'exploitation des racines ont pour désavantage la réduction de la vitalité des arbres, la diminution de la fréquence et de l'intensité des phénomènes phénologiques tels que la floraison et la fructification (Omondi et al., 2016b ; Djekonbe et al., 2018). La plus grande contrainte de gestion liée à l'exploitation du néré dans la région de la Tandjilé est la collecte intensive des fruits et graines qui réduit toutes les chances de régénération naturelle de l'espèce. En effet, la forte demande de ces organes pour la 
consommation des ménages et la commercialisation amène les populations à leur surexploitation. Les pratiques d'exploitation durable devraient donc recommander des quotas de fruits à exploiter par arbre, afin de favoriser la dissémination et la régénération naturelle de l'espèce. Cependant, la connaissance de ces quotas exige des études supplémentaires sur le rendement par tige et les facteurs susceptibles $\mathrm{d}$ 'affecter la production de cette espèce dans les différents systèmes de production (Guedje et al., 2007 ; Maponmetsem et al., 2011).

Le potentiel d'exploitation, de valorisation et de commercialisation des produits du néré est assez élevé dans la zone d'étude. Les résultats non présentés indiquent que la commercialisation des produits de l'espèce rapporte près de $100 \$$ USA $(50000 \mathrm{~F}$ CFA) /mois et représente la principale source de revenus pour les populations de la Tandjilé. Koura et al. (2011) soulignent que les valeurs nutritionnelles et médicinales des produits de $P$. biglobosa leur confèrent un grand potentiel d'utilisation en agroindustrie et pytopharmacie pour la production des arômes et assaisonnements alimentaires, suppléments diététiques et autres phyto-médicaments. Ce fort intérêt comme source d'aliments, de médicaments, de bois de chauffage et de revenus constitue des facteurs susceptibles d'accroitre la demande future des produits et donc la vulnérabilité de l'espèce. Au Tchad comme dans la plupart des pays situés en zone soudano-sahélienne, $P$. biglobosa est classé parmi les cinq premières espèces prioritaires pour la conservation de part leur importance socioéconomique, leur popularité et à cause des multiples pressions auxquelles elles sont soumises.

L'habitat naturel de l'espèce constitué de forêts claires et de savanes herbeuses est connu pour sa haute vulnérabilité aux feux de brousses et à la désertification qui contribue à la dégradation de sa végétation. Les systèmes de production de $P$. biglobosa dans la zone d'étude sont constitués essentiellement des systèmes cultivés (jachères et champs agricoles) et peu de tiges sont exploitées dans les forêts claires. Ce résultat indique que l'espèce est en phase de transition dans la zone entre le statut sauvage et domestiqué. Cependant, la faible représentation des tiges juvéniles dans ces systèmes indique d'une part un faible effort de plantation par les agriculteurs, qui se contenteraient de préserver les arbres lors de la création des plantations et d'autre part des difficultés des jeunes tiges à se maintenir dans cet environnement soumis à de nombreux stress (Raebid et al., 2012). La promotion de la domestication de l'espèce devrait être envisagée, ainsi que l'enrichissement des peuplements à travers des techniques peu coûteuses et adaptées au contexte soudano-sahélien (Bellefontaine et al., 2012) afin de garantir l'approvisionnement futur des ménages face au vieillissement des vergers et à la dégradation des habitats naturels. En effet, le potentiel de domestication de l'espèce dans la région est hautement justifié à travers plusieurs facteurs parmi lesquels :

Son potentiel sur pied plus élevé dans les systèmes cultivés par rapport au système naturel qui traduit l'intérêt des populations pour la production de l'espèce dans leurs systèmes de culture ;

Les bonnes performances en termes de diamètre, hauteur, surface terrière et volumes dans les systèmes cultivés indiquant la compatibilité écologique de l'espèce avec les cultures associées (Bayala et al., 2003) ;

La multiplicité des produits, services et formes d'usages y compris la génération des revenus qui démontrent la capacité de l'espèce à contribuer au bienêtre des populations ;

Les multiples pressions exercées sur les peuplements ajoutées aux contraintes de gestion durable citées par les agriculteurs soulèvent la nécessité de préserver et intensifier les systèmes de production actuels et de créer des sources alternatives d'approvisionnement.

Cependant, le processus de domestication de l'espèce exigera des études supplémentaires sur : 
La documentation de tous les niveaux de connaissances sur l'espèce afin de mieux apprécier son potentiel de valorisation dans le pays ;

L'analyse de l'état actuel de la régénération naturelle et des exigences écologiques de croissance et de développement des plantules dans les différents systèmes de production agricole existants ;

L'identification des pratiques sylvicoles les plus appropriées pour maintenir une bonne productivité des arbres, limiter les interactions négatives et accroître l'effet positif de l'arbre à néré sur la production des cultures associées ;

La caractérisation et l'identification des morphotypes supérieurs qui pourraient être multipliés végétativement par bouturage sous châssis de propagation contribuant ainsi à améliorer quantitativement et qualitativement les populations existantes du néré au Tchad à travers la domestication.

\section{Conclusion}

$\mathrm{Au}$ terme de cette étude sur l'évaluation $\mathrm{du}$ potentiel écologique et ethnobotanique du néré dans la région de Tandjile-Ouest au Sud du Tchad, il a été note que $P$. biglobosa prédomine dans les systèmes agricoles cultivés et dans les jachères, mais sont sous représentés dans les forêts claires. La régénération de cette espèce est quasiabsence due à des perturbations dans la dynamique des tiges d'avenir dans les agrosystèmes, traduisant ainsi son statut vulnérable dans la région. Du point de vue ethnobotanique, la graine et la pulpe des fruits sont les plus utilisés avec des valeurs d'usage essentiellement alimentaires et commerciales. La surexploitation des fruits dans les systèmes cultivés et la carbonisation des forêts sont les principales contraintes à la gestion durable de cette espèce au Sud du Tchad. La domestication de l'espèce est ainsi recommandée comme une stratégie pouvant permettre d'accroitre la régénération dans les systèmes cultivés et d'intensifier la production, d'autre part la sensibilisation des populations aux techniques rationnelles d'exploitation des organes afin de réduire la pression sur les peuplements et assurer la pérennisation des ressources de l'espèce dans la région. Il serait judicieux que cette étude s'étende à d'autres espèces ligneuses prioritaires afin d'élaborer des stratégies nationales raisonnées pour la gestion durable des espèces ligneuses ayant des intérêts socioéconomiques remarquables.

\section{CONFLIT D'INTERETS}

Les auteurs déclarent qu'il n'y a aucun conflit d'intérêts.

\section{CONTRIBUTIONS DES AUTEURS}

MLAA-T et SK ont conçu le thème, élaboré le protocole de recherche. SK a collecté les données sur le terrain avec l'assistance technique de BMM. MLAA-T a contribué à l'analyse des données qui ont permis à SK de proposer la première mouture du manuscrit. Le manuscrit a été retravaillé par DDN. MLAA-T a finalisé et produit la dernière mouture du manuscrit qui a été relu et validé par tous les co-auteurs.

\section{REMERCIEMENTS}

Les auteurs remercient la Direction des Forêts du Tchad pour l'appui logistique apporté pendant la collecte des données, ainsi que toutes les populations de la région de Tandjilé pour leur disponibilité à participer à l'étude.

\section{REFERENCES}

Abdourhamane H, Rabiou H, Diouf A, Morou B, Mahamane A et Bellefontaine R. 2017. Structure démographique et répartition spatiale des populations de Sclerocarya birrea (A. Rich.) Hochst. du secteur sahélien du Niger. Bois et Forêts des Tropiques, 333(3) : 55-77. DOI: 10.19182/bft2017.333.a31468

Azokpota P, Hounhouigan DJ, Nago MC. 2006. Microbiological and chemical changes during the fermentation of African locust bean (Parkia biglobosa) to produce afitin, iru and sonru, three 
traditional condiments produced in Benin. International Journal of Food Microbiology, 107: 304-309. DOI: https://doi.org/10.1016/j.ijfoodmicro.200 5.10 .026

Bayala J, Mando A, Ouedraogo SJ, Teklehaimandot Z. 2003. Managing Parkia biglobosa and Vitellaria paradoxa Prunings for Crop Production and Improved Soil Properties in the SubSudanian Zone of Burkina Faso. Arid Land Research and Management, 17(3): 283-296.

DOI: https://doi.org/10.1080/15324980301596

Bellefontaine R, Malagnoux M, Ichaou A. 2012. Techniques forestières et innovations dans les opérations de reboisement. In: La Grande Muraille Verte: Capitalisation des recherches et valorisation de savoirs locaux Dia A, Duponnois R (éds); Marseille, France, IRD Éditions, 493 p. (+ cédérom).

Betti JL, Yemefa'a SRM, Tarla FN. 2011. Contribution to the knowledge of nonwood forest products of the far north region of Cameroon: Medicinal plants sold in the Kousseri market. Journal of Ecology and the Natural Environment, 3(7):241-254.

http://www.academicjournals.org/jene

Blench R. 2003. Arbres fruitiers sauvages et protégés dans la préfecture du Logone Oriental, sud du Tchad. Overseas Development Institute Londres, 18 pages

Chupezi TJ, Ndoye O, Tchatat M, Chikamai B. 2009. Processing and Marketing of Non-wood Forest Products: Potential Impacts and Challenges in Africa. Discovery \& Innovations, 21(SFM Special Edition No. 1): 60-65.

Codjia JTC, Assogbadjo AE, Ekué MRM. 2003. Diversité et valorisation au niveau local des ressources végétales forestières alimentaires du Bénin. Cahiers Agricultures, 12 : 1-12.

Dicko A, Biaou HSS, Natta AK, Gouwakinnou G. 2017. Quantitative ethnobotany of Lophira lanceolata Tiegh. ex Keay (Ochnaceae) in Benin (West Africa). Int. J. Biol. Chem. Sci., 11(3):
1236-1253.

DOI:

https://dx.doi.org/10.4314/ijbcs.v11i3.25

Dongock ND, Avana-Tientcheu ML, Mbaiti Djimasngar, Saradoum Goy, Pinta JY. 2017. Importance écologique et potentialité apicole à la périphérie du Parc national de Manda en zone soudanienne du Moyen-Chari (Tchad). International Journal of Environmental Studies, 74(3): 443-457. DOI: 10.1080/00207233.2017.1294424.

Djekonbe P, Avana-Tientcheu ML, Womeni HM. 2018. Influence des attaques des Loranthaceae et des pressions anthropiques sur la structure des populations de Vitellaria paradoxa au Tchad. Revue Forêts et Environnement du Bassin du Congo, 11: 39-48. DOI : http://dx.doi.org/10.5281/zenodo.143721 0

Eba' AR, Lescuyer G, Gouhouo PJ, Moulende FT. 2013. Etude de l'importance économique et sociale du secteur forestier et faunique dans les Etats d'Afrique Centrale: Cas du Cameroun, Rapport d'étude CIFOR, 316p.

FAO. 2009. The state of Security in the World. Agricultural Organization of the United Nations Rome. 180 pages.

FAO. 2014. State of the World's Forests (SOFO). Enhancing the socioeconomic benefits from forests: Putting people at the centre. ISSN 1020-5705. 133p

Guedje NM, Fokunang NC, Jiofack TRB, Dongmo FR. 2010. Opportunités d'une exploitation soutenue des plantes médicinales dans l'aménagement forestier. Int. J. Biol. Chem. Sci., 4(4): 1346-1372. http://ajol.info/index.php/ijbcs

Guedje NM, Zuidema PA, During H, Foahom B, Lejoly J. 2007. Tree bark as a nontimber forest product: The effect of bark collection on population structure and dynamics of Garcinia lucida Vesque. Fo. Ecol. Man., 240(1-3) : 1-12. DOI : https://doi.org/10.1016/j.foreco.2006.09. 029

Goudiaby M. 2014. Le fruit du néré comme moyen de lutter contre la pauvreté des 
ménages au Sénégal. Sahel Agroforesterie, 20 : 2-3.

Houehanou DT, Assogbadjo AE, Chadare FJ, Zanvo S, Sinsin B. 2016. Approches méthodologiques synthétisées des études d'ethnobotanique quantitative en milieu tropical. Annales des Sciences Agronomiques du Bénin, 20 (numéro Spécial) : 187-205.

Komolafe K, Olaleye TM, Omotuyi, OI, Boligon AA, Athayde ML, Akindahunsi AA, Da Rocha JBT. 2014. Parkia biglobosa Improves Mitochondrial Functioning and Protects against Neurotoxic Agents in Rat Brain Hippocampal Slices. Journal of Biomedicine and Biotechnology, 3: 15p. DOI: $10.1155 / 2014 / 326290$

Koura K, Ganglo JC, Assogbadjo AE, Agbangla C. 2011. Ethnic differences in use values and use patterns of Parkia biglobosa in Northern Benin. Journal of Ethnobiology and Ethnomedecine, 7: 42. http://www.ethnobiomed.com/content/7/ $1 / 42$

Koura K, Mbaide Y, Ganglo JC. 2013. Caractéristiques phénotypique et structurale de la population de Parkia biglobosa (Jacq.) du Nord-Bénin. Int. J. Biol. Chem. Sci., 7(6): 2409-2425. DOI: http://dx.doi.org/10.4314/ijbcs.v7i6.19

Maazou R, Rabiou H, Issiaka Y, Abdou L, Saidou IS, Mahamane A. 2017. Influence de l'occupation des terres sur la dynamique des communautés végétales en zone Sahélienne : cas de la commune rurale de Dantchandou (Niger). Int. J. Biol. Chem. Sci., 11(1): 79-92. DOI: http://dx.doi.org/10.4314/ijbcs.v11i1.7

Maisharou A, Larwanou M. 2015. Market Potential of Non-Wood Forest Products in The Sahelian Countries. International Journal of Forestry Review, 17(S3): 125136.

Mapongmetsem PM, Dona A. 2010. Land use systems biodiversity conservation East Tandjile, Chad. Scripta Bot. Belg., 46: 285.
Mapongmetsem PM, Nkongmeneck BA, Rongoumi G, Dongock DN, Dongmo B. 2011. Impact des systèmes d'utilisation des terres sur la conservation de Vitellaria paradoxa Gaerten. F. (Sapotaceae) dans la région des savanes soudano-guinéennes. International Journal of Environmental Studies, 68(6): 851-872.

DOI : https://doi.org/10.1080/00207233.2011.5 87259

Maréchal C, Cawoy V, Coquyt C, Dauby G, Dessein S, Douglas-Hamilton I, Dupain J, Fischer E, Obang DF, Groom Q, Henshel P, Jeffery KJ, Korte L, Lewis SL, Lubunu S, Maisels F, Melletti M, Ngouffo R, Ntore S, Palla F, Scholte P, Sonke B, Stevart T, Stoffelen P, Van Den Broeck D, Walters G, Williamson EA. 2014. Conservation et Gestion de la biodiversité. In Etat des Forets 2013, De Wasseige C, Flynn J, Louppe D, Hiol Hiol F, Mayaux Ph (eds). WeyRich: Belgique ; 67-96.

Millogo-Kone H, Lompo M, Kini F, Asimi S, Guissou IP, Nacoulma O. 2009. Evaluation of flavonoids and total phenolic contents of stem bark and leaves of Parkia biglobosa (Jacq.) Benth. (Mimosaceae)-free radical scavenging and antimicrobial activities. Research Journal of Medical Sciences, 3(2): 7074.http://medwelljournals.com/abstract/? doi=rjmsci.2009.70.74.

Moulnang T, Kadiom A. 2001. Situation des ressources génétiques forestières $\mathrm{du}$ Tchad. Département des forêts, FAO. Consulté le 07 Mars 2013 www.fao.org.

Nchoutpouen C, Mapongmetsem PM, Zapfack L, Ngo Peck ML. 2009. Effect of land use systems on structure and population of Parkia biglobosa in the Sudano-guinean savannah. Forests, Trees and Livelihoods, 19: 69-79.

Odebiyi JA, Bada SO, Awodoyin RO, Oni PI, Omoloye AA. 2004. Population strucrure of Vitelaria Paradoxa Gaertn. and Parkia biglobosa (Jacq) Benth. in the Agroforestry Parlands of Nigerian humid savanna. West African Journal of applied 
Ecology, 5: 31-39. DOI: 10.4314/wajae.v5i1.45597.

Padakale E, Atakpama W, Dourma M, Dimobe K, Wala K, Guelly KA, Akpagana K. 2015, Woody Species Diversity and Structure of Parkia biglobosa Jacq. Dong Parklands in the Sudanian Zone of Togo (West Africa). Annual Research \& Review in Biology, 6(2): $\quad$ 103-114. DOI: 10.9734/ARRB/2015/14105

Ræbild A, Hansen UB, Kambou S. 2012. Regeneration of Vitellaria paradoxa and Parkia biglobosa in a parkland in Southern Burkina Faso. Agroforestry Systems, 85: 443-453. DOI: 10.1007/s10457-011-9397-0.

Reyes-Garcia V, Byron E, Godoy VVR, Apaza L, Limache EP, Leonard WR, Wilkie D: Measuring culture as shared knowledge: do data collection formats matter? Cultural knowledge of plants uses among Tsimane' Amerindians, Bolivie. Field Methods, 16(2): 135-156.

Omondi SF, Odee DW, Ongamo GO, Kanya JI, Khasa DP. 2016a. Genetic consequences of anthropogenic disturbances and population fragmentation in Acacia Senegal. Conservation Genetics, 17: 1235 -1244. DOI : https://doi.org/10.1007/s10592016-0854-1.

Omondi SF, Odee DW, Ongamo GO, Kanya JI, Khasa DP. 2016b. Synchrony in Leafing, Flowering, and Fruiting Phenology of Acacia senegal within Lake Baringo Woodland, Kenya: Implication for Conservation and Tree Improvement. International Journal of Forestry Research: 11 pages. http://dx.doi.org/10.1155/2016/6904834. 